\title{
Um Estudo Empírico Sobre o Impacto Macroeconômico do Programa Bolsa Família
}

\author{
Wellington Furtado ${ }^{1}$ \\ Glauco Oliveira Rodrigues ${ }^{2}$ \\ Eugenio de Oliveira Simonetto ${ }^{3}$ \\ Daniel Visentini de Barcellos ${ }^{4}$
}

http://dx.doi.org/10.21527/2237-6453.2017.39.68-85

\begin{abstract}
Resumo
0 presente estudo busca identificar o impacto que o Programa Bolsa Família (PBF) tem sobre 0 desenvolvimento econômico nas cinco regiões do Brasil, tendo como proxy para o desenvolvimento regional o consumo de energia elétrica residencial. Com o constante aumento no número de famílias beneficiadas e recursos disponíveis para o PBF, verificamos a relação existente entre a bolsa recebida pelas famílias e o consumo de bens. Este estudo se justifica pelo interesse no desenvolvimento econômico brasileiro e apresenta qual o impacto socioeconômico que, coletivamente, os beneficiários do programa causam.
\end{abstract}

Palavras-chave: Programa Bolsa Família. Consumo. Desenvolvimento econômico.

\footnotetext{
Doutorando em Administração pela Universidade Federal de Santa Maria (UFSM). Mestre em Economia Empresarial pela Universidade Cândido Mendes do Rio de Janeiro. Graduado em Administração de Empresas pelas Faculdades Integradas Simonsen. Professor do Instituto Federal Farroupilha, campus São Vicente do Sul-RS. wellington.furtado@gmail.com

2 Mestre em Administração pela Universidade Federal de Santa maria, Graduado em Redes de Computadores pela Universidade Federal de Santa Maria. glaucop10@redes.ufsm.br

3 Doutor em Administração pela Universidade Federal do Rio Grande do Sul (UFRGS). Mestre em Ciência da Computação pela Pontifícia Universidade Católica do Rio Grande do Sul (PUCRS). Graduado em Análise de Sistemas pela Universidade Católica de Pelotas (Ucpel). Professor da Universidade Federal de Santa Maria (UFSM). eosimonetto@gmail.com

'Graduado em Redes de Computadores pela Universidade Federal de Santa Maria, Técnico da Tecnologia da Informação na Universidade Federal de Santa Maria, danielvisentini@ufsm.br.

4 Graduado em Redes de Computadores pela Universidade Federal de Santa Maria. Técnico da Tecnologia da Informação na Universidade Federal de Santa Maria, danielvisentini@ufsm.br.
} 


\section{AN EMPIRICAL STUDY ON THE MACROECONOMIC IMPACT OF THE BOLSA FAMÍLIA PROGRAM}

\section{Abstract}

This study sought to identify the impact of the Bolsa Família Program (BFP) has on economic development in five regions of Brazil, with the proxy para regional development residential electricity consumption. With the steady increase in the number of beneficiary families and the resources available to GMP, we find the relationship between the scholarship received by households and consumer goods. This study is justified by the interest in the Brazilian economic development and presents which the socio-economic impact that collectively cause the program's beneficiaries.

Keywords: Family Allowance. Consumption. Economic Development. 
O Programa Bolsa Família, criado no governo Lula em 2003, unificou o programa Fome Zero e os programas que surgiram no governo Fernando Henrique Cardoso (Bolsa Escola, Bolsa Alimentação, Cartão Alimentação e Auxílio Gás). Esses programas objetivam a transferência de renda baseada na ajuda financeira às famílias pobres, que possuem renda per capita de 70 até 140 reais, e extremamente pobres, com renda per capita menor que 70 reais. A contrapartida das famílias beneficiárias é que mantenham seus filhos e/ou dependentes com idade até 7 anos com frequência regular na escola e devidamente vacinados, seguindo o calendário básico de vacinação da criança do Ministério da Saúde.

O Bolsa Família integra o Plano Brasil Sem Miséria (BSM), que tem por objetivo alcançar os 16 milhões de brasileiros com renda familiar per capita inferior a $\mathrm{R} \$ 70$ mensais, e está baseado na garantia de renda, inclusão no processo produtivo e acesso aos serviços públicos, como os serviços básicos de educação e saúde.

Atualmente o Programa Bolsa Família é considerado o principal programa de transferência de renda existente no Brasil. Ele possui elevada alocação e beneficia, atualmente, cerca de 13 milhões de famílias, com um investimento realizado em torno de $\mathrm{R} \$ 12$ bilhões (MINISTÉRIO..., 2011).

O Programa Bolsa Família caracteriza-se como estratégia de ruptura do ciclo da pobreza, quando as famílias passam a ter um complemento de renda. Para evitar a duplicidade de ações entre os governos federal e municipal, a União utiliza algumas estratégias, como a possibilidade de integrar programas municipais de renda com o Programa Bolsa Família, a adesão formal dos municípios ao Programa Bolsa Família e o estabelecimento de processos sistêmicos de apoio financeiro para a gestão estadual e local (MEDEIROS; BRITTO; SOARES, 2007).

Como principal programa de inclusão e promoção do desenvolvimento social do país, ele vem sendo analisado pelo governo e também por acadêmicos, organismos e sociedade civil, por sua capacidade em promover um 
impacto econômico na vida das famílias que o recebem, além da elevação do consumo no curto prazo, o combate e a redução da pobreza, e a melhora dos padrões de saúde e educação (SANTOS; SANTOS, 2007).

Com base nos dados da Pesquisa Nacional por Amostra de Domicílio (PNAD), realizada pelo Instituto Brasileiro de Geografia e Estatística (IBGE), a proporção de brasileiros com renda inferior a um dólar por dia era de 8,8\% em 1990 e foi reduzida a 4,2\% em 2005, e apenas o Programa Bolsa Família contribuiu diretamente em $21 \%$ para a redução da pobreza no Brasil. Alguns estudos apresentam análises identificando como principal fator de alteração a estabilidade da moeda, a diminuição do desemprego e os Programas de Transferência de Renda (GUSMÃO, 2009).

Levando em consideração a importância do programa, seu caráter inédito e o alto dispêndio realizado, é fundamental que seus impactos sejam avaliados. Este artigo propõe-se a verificar se o consumo de energia elétrica industrial e residencial foi afetado pelo dispêndio com o programa. Tal análise justifica-se pelo fato de que o consumo de energia elétrica é, tradicionalmente, uma proxy para o desenvolvimento econômico em âmbito macro.

A proposta é identificar a possibilidade de mudança socioeconômica nos beneficiados do Programa Bolsa Família, apresentando resultados que mostram a importância da inclusão que essas famílias precisam para sair de um status quo e ter acesso a uma vida mais digna e promissora, tendo em vista que muitas mudanças são ainda necessárias para chegar a essa realidade, mas torna-se necessário programas com essa finalidade para auxiliar nessa mudança social e econômica.

\section{Aspectos Teóricos e Estudos Anteriores}

Licio, Mesquita e Curralero (2011) inserem-se na temática do federalismo e das relações intergovernamentais e políticas, cujo principal enfoque consiste no Programa Bolsa Família qualificar o debate sobre a centralização 
dos programas de transferência de renda no Brasil por meio da análise das relações intergovernamentais adotadas no âmbito das três principais dimensões: transferência de renda, acompanhamento de condicionalidades e articulação de programas complementares - considerando-se as implicações federativas derivadas da perspectiva intersetorial que rege o programa. Outro tema debatido pelos autores é a questão intergovernamental que demanda a organização de uma estratégia nacional coordenada de articulação de programas complementares, sugerindo-se maior participação dos Estados na coordenação regional do Programa.

Hoffmann (2010), utilizando os dados da Pesquisa de Orçamentos Familiares (POF) de 2008-2009, analisou as principais características da distribuição da renda familiar per capita (RFPC) no Brasil e em seis regiões, comparando com resultados obtidos da POF de 2002-2003, e a distribuição da despesa total per capita. A RFPC foi calculada para cada família, dividindo o valor do seu rendimento total e a variação patrimonial pelo número de pessoas da família. De 2001 a 2009 chegou a um processo sistemático de redução da desigualdade, com o índice de Gini da distribuição do rendimento domiciliar per capita caindo de 0,594 para 0,539 .

Melo e Duarte (2010) avaliam o impacto do Programa Bolsa Família sobre a frequência escolar de crianças e adolescentes de 5 a 14 anos na agricultura familiar dos Estados de Pernambuco, Ceará, Sergipe e Paraíba. Nessa investigação, os autores utilizam dados primários (pesquisa de campo) e dados secundários (BRASIL, 2005) para obter estimativas de propensity score. Os resultados indicam que, de forma geral, o programa eleva a frequência escolar das referidas crianças no intervalo de 5,4 a 5,9 pontos porcentuais. Apesar disso, há importantes diferenças quando se considera meninas e meninos separadamente, sendo o programa eficaz no primeiro caso e ineficaz no segundo, ou seja, não parece haver efeito do programa sobre a frequência escolar dos meninos, o que pode estar associado a diferenças de gênero nos custos de oportunidades do investimento em capital humano no meio rural. 
Rocha (2011) faz um breve retrospecto analisando a recente evolução do Programa Bolsa Família no que diz respeito à focalização e cobertura da população-alvo, utilizando informações dos PNADs 2004 e 2006. Apesar de o programa ter atingido a meta de 11 milhões de domicílios no final de 2006, há evidências de que cerca de 3,4 milhões de domicílios elegíveis ainda se encontram a descoberto. Simulações evidenciam que garantir a cobertura de todos os domicílios elegíveis tem maior impacto sobre os indicadores de pobreza do que conceder um novo benefício.

Santos et al. (2010) analisam a entrada de homens e mulheres no mercado de trabalho rural e geram equações de rendimento para o meio rural brasileiro em 2007 com os microdados da PNAD. O procedimento de Heckman é adotado para se evitar viés de seletividade. Os resultados mostraram gaps na seleção e rendimentos por gênero e raça. Apesar de as mulheres apresentarem um grau de qualificação maior que o dos homens, observa-se que o rendimento destes tende a ser mais elevado do que o feminino. Adicionalmente, mulheres contempladas com o Bolsa Família tendem a reduzir a oferta de trabalho.

Rego (2007) busca recordar, de forma sucinta, a história e a teoria dos direitos e da cidadania, procurando que façam parte de seu inventário temático. Busca, ainda, demonstrá-las por meio de visões do mais amplo interesse pelas relações Estado e sociedade, participação política, mercado e justiça distributiva, e a inter-relação entre essas variáveis com a questão democrática, efetuando um balanço crítico na bibliografia sobre a questão.

Moura (2007) apresenta uma análise crítica do Programa Bolsa Família, instituído pelo governo Lula, a partir da metodologia de ongoing analysis conjuntural, buscando estabelecer um paralelo dessas análises com as críticas sobre o Programa Bolsa Família, que, por meio de dados secundários, norteiam as políticas sociais de governo, sugerindo que a solução posta pelo governo foi uma resposta conjuntural a uma demanda de marketing governamental, e não orientada por uma política pública de governo. 


\section{Metodologia}

O presente artigo caracteriza-se como uma pesquisa de caráter exploratório, baseada em dados quantitativos. No intuito de cumprir com o objetivo proposto, foi realizado o levantamento das despesas com o Programa Bolsa Família (PBF) em todos os Estados da União entre outubro de 2003 e outubro de 2010. Os dados são mensurados em Reais (\$), de frequência mensal e foram obtidos junto ao Ministério do Desenvolvimento Social (MDS). Os dados acerca do consumo de energia elétrica residencial e industrial foram obtidos junto ao IBGE por macrorregião geográfica mensurados em KWh (quilowatt/hora), em frequência mensal, referentes ao mesmo período em que os demais.

Para que as variáveis se tornassem análogas, foi necessário somar o dispêndio com o PBF por região, pois a disponibilidade de dados acerca do consumo de energia elétrica é apenas regional. Além das variáveis regionais, trabalhou-se também com variáveis que representam o contexto nacional, formadas a partir da soma dos valores regionais.

Antes de proceder à análise dos dados foi verificada a estacionaridade das séries mediante o teste de Dickey-Fuller Aumentado (ADF). A hipótese nula de raiz unitária não foi rejeitada para nenhuma variável, ao grau de significância de 5\%. Em seguida, foi calculada a primeira diferença de cada uma e o teste foi realizado novamente, e desta vez a hipótese nula de raiz unitária foi rejeitada. Ou seja, a primeira diferença das séries pode ser considerada estacionária. Dessa forma, as variáveis analisadas neste estudo são dispostas no Quadro 1.

Quadro 1 - Variáveis utilizadas neste estudo

\begin{tabular}{lcc}
\hline Variável & $\begin{array}{c}\text { Símbo- } \\
\text { lo }\end{array}$ & $\begin{array}{c}\text { Unidade } \\
\text { de Medida }\end{array}$ \\
\hline $\begin{array}{l}\text { Primeira Diferença do dispêndio com o PBF no Brasil } \\
\text { Primeira Diferença do dispêndio com o PBF na Região }\end{array}$ & $\mathrm{x}_{(\mathrm{br})}$ & Reais $(\$)$ \\
$\begin{array}{l}\text { Centro-Oeste } \\
\mathrm{x}_{(\mathrm{co})}\end{array}$ & Reais $(\$)$
\end{tabular}


Primeira Diferença do dispêndio com o PBF na Região Nordeste

Primeira Diferença do dispêndio com o PBF na Região Norte

Primeira Diferença do dispêndio com o PBF na Região Sudeste

Primeira Diferença do dispêndio com o PBF na Região Sul

Primeira Diferença do consumo de energia elétrica residencial no Brasil

Primeira Diferença do consumo de energia elétrica residencial na Região Centro-Oeste

Primeira Diferença do consumo de energia elétrica residencial na Região Nordeste

Primeira Diferença do consumo de energia elétrica residencial na Região Norte

Primeira Diferença do consumo de energia elétrica residencial na Região Sudeste

Primeira Diferença do consumo de energia elétrica residencial na Região Sul

Primeira Diferença do consumo de energia elétrica industrial no Brasil

Primeira Diferença do consumo de energia elétrica industrial na Região Centro-Oeste

Primeira Diferença do consumo de energia elétrica industrial na Região Nordeste

Primeira Diferença do consumo de energia elétrica industrial na Região Norte

Primeira Diferença do consumo de energia elétrica industrial na Região Sudeste

Primeira Diferença do consumo de energia elétrica industrial na Região Sul
$X_{(n e)}$

Reais (\$)

Reais (\$)

Reais (\$)

Reais (\$)

KWh

KWh

KWh

KWh

KWh

KWh

KWh

KWh

$\mathrm{y}_{\text {(ine) }} \quad \mathrm{KWh}$

$\mathrm{y}_{(\text {in) }} \quad \mathrm{KWh}$

$\mathrm{y}_{\text {(ise) }} \quad \mathrm{KWh}$

$\mathrm{y}_{(\text {is) }} \quad \mathrm{KWh}$

Fonte: Elaborado pelos autores.

Tendo definidas as variáveis a serem analisadas, serão estimadas as estatísticas descritivas das séries em questão, e, em seguida, regressões lineares para analisar o relacionamento entre as variáveis. O objetivo deste 
estudo é averiguar o impacto macroeconômico do dispêndio com o PBF; para isso considerou-se as variáveis de consumo de energia elétrica como dependentes e as variáveis de dispêndio com PBF independentes. Para melhorar a qualidade da análise foi inserido um vetor autorregressivo em cada equação, além do quadrado da variável independente, amenizando os possíveis problemas com autocorrelação e heteroscedasticidade. Dessa forma, as análises realizadas neste estudo podem ser resumidas pela Equação [01].

$$
y_{i, t}=\alpha+\beta_{1} y_{i, t-1}+\beta_{2} x_{i, t}+\beta_{3} x_{i, t}^{2}+\varepsilon_{t} \quad[01],
$$

em que representa a variação do consumo de energia elétrica da região $i$ no período $t ; x_{i, t}$ representa a variação do dispêndio com o PBF na região $i$ no período $t, \beta_{1}, \beta_{2}, \beta_{3}$, e são parâmetros; $\alpha$ é uma constante e $\varepsilon_{t}$ representa o erro da estimação no período $t$.

A Equação [01] será aplicada para verificar o relacionamento entre a variação do consumo de energia elétrica residencial e o dispêndio com PBF nas cinco macrorregiões geográficas e no Brasil. Em seguida, o procedimento será repetido substituindo a variação do consumo de energia elétrica residencial pela industrial, gerando um total de 12 regressões lineares.

Por fim, o teste de causalidade de Granger será aplicado para verificar se há evidências de que existem relações de causalidade entre as variáveis analisadas. A seção a seguir apresentará os resultados obtidos.

\section{Resultados}

Para dar início à apresentação dos resultados, a Tabela 1 traz as estatísticas do teste ADF.

Tabela 1 - Teste de Dickey-Fuller Aumentado

\begin{tabular}{lc|c}
\hline Variável & $\begin{array}{c}\text { Estatística } \\
\text { de Teste }\end{array}$ & $\begin{array}{c}\text { P-valor } \\
\text { (MacKinnon) }\end{array}$ \\
\hline $\mathrm{x}(\mathrm{br})$ & $-8,4150$ & 0,0000
\end{tabular}




\begin{tabular}{lr|l}
$\mathrm{x}(\mathrm{co})$ & $-8,3300$ & 0,0000 \\
$\mathrm{x}(\mathrm{ne})$ & $-8,4370$ & 0,0000 \\
$\mathrm{x}(\mathrm{n})$ & $-7,6500$ & 0,0000 \\
$\mathrm{x}(\mathrm{se})$ & $-8,8610$ & 0,0000 \\
$\mathrm{x}(\mathrm{s})$ & $-8,3370$ & 0,0000 \\
$\mathrm{y}(\mathrm{rbr})$ & $-12,4840$ & 0,0000 \\
$\mathrm{y}(\mathrm{rco})$ & $-11,0760$ & 0,0000 \\
$\mathrm{y}(\mathrm{rne})$ & $-9,5460$ & 0,0000 \\
$\mathrm{y}(\mathrm{rn})$ & $-8,3420$ & 0,0000 \\
$\mathrm{y}(\mathrm{rse})$ & $-12,7820$ & 0,0000 \\
$\mathrm{y}(\mathrm{rs})$ & $-11,3270$ & 0,0000 \\
$\mathrm{y}(\mathrm{ibr})$ & $-6,7570$ & 0,0000 \\
$\mathrm{y}(\mathrm{ico})$ & $-9,5430$ & 0,0000 \\
$\mathrm{y}(\mathrm{ine})$ & $-14,2300$ & 0,0000 \\
$\mathrm{y}($ in $)$ & $-14,9440$ & 0,0000 \\
$\mathrm{y}$ (ise) & $-7,5520$ & 0,0000 \\
$\mathrm{y}($ is $)$ & $-9,1830$ & 0,0000 \\
\hline
\end{tabular}

Fonte: Elaborado pelos autores.

Após o cálculo da primeira diferença, o teste ADF rejeitou a hipótese nula de raiz unitária para todas as variáveis analisadas ao grau de significância de 5\%, conforme já descrito na seção anterior. A Tabela 2 expõe as estatísticas descritivas para as variáveis diferenciadas.

Tabela 2 - Estatísticas Descritivas

\begin{tabular}{lccccccc}
\hline Variável & Média & Mediana & Mínimo & Máximo & $\begin{array}{c}\text { Desvio- } \\
\text {-Padrão }\end{array}$ & $\begin{array}{c}\text { Enviesa- } \\
\text { mento }\end{array}$ & $\begin{array}{c}\text { Curtose } \\
\text { Ex. }\end{array}$ \\
x(br) & $1,21 \mathrm{E}+07$ & $8,03 \mathrm{E}+06$ & $-3,61 \mathrm{E}+07$ & $1,27 \mathrm{E}+08$ & $2,50 \mathrm{E}+07$ & $2,63 \mathrm{E}+00$ & $8,80 \mathrm{E}+00$ \\
$\mathrm{x}(\mathrm{co})$ & $6,82 \mathrm{E}+05$ & $2,60 \mathrm{E}+05$ & $-1,52 \mathrm{E}+06$ & $7,90 \mathrm{E}+06$ & $1,47 \mathrm{E}+06$ & $2,60 \mathrm{E}+00$ & $8,67 \mathrm{E}+00$ \\
$\mathrm{x}(\mathrm{ne})$ & $6,07 \mathrm{E}+06$ & $3,35 \mathrm{E}+06$ & $-2,00 \mathrm{E}+07$ & $6,55 \mathrm{E}+07$ & $1,26 \mathrm{E}+07$ & $2,85 \mathrm{E}+00$ & $9,97 \mathrm{E}+00$ \\
$\mathrm{x}(\mathrm{n})$ & $1,53 \mathrm{E}+06$ & $9,12 \mathrm{E}+05$ & $-2,92 \mathrm{E}+06$ & $1,36 \mathrm{E}+07$ & $2,68 \mathrm{E}+06$ & $2,80 \mathrm{E}+00$ & $9,01 \mathrm{E}+00$
\end{tabular}




\begin{tabular}{llllllll}
$\mathrm{x}(\mathrm{se})$ & $2,94 \mathrm{E}+06$ & $1,65 \mathrm{E}+06$ & $-9,28 \mathrm{E}+06$ & $3,15 \mathrm{E}+07$ & $6,81 \mathrm{E}+06$ & $1,87 \mathrm{E}+00$ & $5,38 \mathrm{E}+00$ \\
$\mathrm{x}(\mathrm{s})$ & $9,00 \mathrm{E}+05$ & $1,87 \mathrm{E}+05$ & $-2,73 \mathrm{E}+06$ & $1,02 \mathrm{E}+07$ & $2,30 \mathrm{E}+06$ & $1,66 \mathrm{E}+00$ & $3,42 \mathrm{E}+00$ \\
$\mathrm{y}(\mathrm{rbr})$ & $2,68 \mathrm{E}+04$ & $4,71 \mathrm{E}+04$ & $-4,55 \mathrm{E}+05$ & $6,62 \mathrm{E}+05$ & $2,39 \mathrm{E}+05$ & $-3,65 \mathrm{E}-02$ & $-4,26 \mathrm{E}-01$ \\
$\mathrm{y}(\mathrm{rco})$ & $2,65 \mathrm{E}+03$ & $4,26 \mathrm{E}+03$ & $-5,02 \mathrm{E}+04$ & $4,53 \mathrm{E}+04$ & $2,30 \mathrm{E}+04$ & $-1,80 \mathrm{E}-01$ & $-6,10 \mathrm{E}-01$ \\
$\mathrm{y}(\mathrm{rne})$ & $6,30 \mathrm{E}+03$ & $8,22 \mathrm{E}+03$ & $-9,84 \mathrm{E}+04$ & $1,50 \mathrm{E}+05$ & $4,49 \mathrm{E}+04$ & $1,90 \mathrm{E}-01$ & $5,82 \mathrm{E}-02$ \\
$\mathrm{y}(\mathrm{rn})$ & $2,38 \mathrm{E}+03$ & $1,75 \mathrm{E}+03$ & $-3,76 \mathrm{E}+04$ & $3,78 \mathrm{E}+04$ & $1,43 \mathrm{E}+04$ & $-1,30 \mathrm{E}-01$ & $-1,56 \mathrm{E}-01$ \\
$\mathrm{y}(\mathrm{rse})$ & $1,27 \mathrm{E}+04$ & $1,20 \mathrm{E}+04$ & $-2,77 \mathrm{E}+05$ & $4,26 \mathrm{E}+05$ & $1,51 \mathrm{E}+05$ & $1,31 \mathrm{E}-01$ & $-3,44 \mathrm{E}-01$ \\
$\mathrm{y}(\mathrm{rs})$ & $2,77 \mathrm{E}+03$ & $-2,10 \mathrm{E}+03$ & $-9,38 \mathrm{E}+04$ & $1,80 \mathrm{E}+05$ & $5,28 \mathrm{E}+04$ & $9,46 \mathrm{E}-01$ & $1,69 \mathrm{E}+00$ \\
$\mathrm{y}(\mathrm{ibr})$ & $4,45 \mathrm{E}+04$ & $1,11 \mathrm{E}+05$ & $-1,49 \mathrm{E}+06$ & $7,12 \mathrm{E}+05$ & $3,92 \mathrm{E}+05$ & $-1,64 \mathrm{E}+00$ & $4,15 \mathrm{E}+00$ \\
$\mathrm{y}$ (ico) & $2,33 \mathrm{E}+03$ & $4,02 \mathrm{E}+03$ & $-5,56 \mathrm{E}+04$ & $7,18 \mathrm{E}+04$ & $2,47 \mathrm{E}+04$ & $1,60 \mathrm{E}-02$ & $4,26 \mathrm{E}-02$ \\
$\mathrm{y}($ ine $)$ & $6,90 \mathrm{E}+03$ & $1,02 \mathrm{E}+03$ & $-1,95 \mathrm{E}+05$ & $2,26 \mathrm{E}+05$ & $8,90 \mathrm{E}+04$ & $2,16 \mathrm{E}-01$ & $-2,11 \mathrm{E}-01$ \\
$\mathrm{y}(\mathrm{in})$ & $3,87 \mathrm{E}+03$ & $6,81 \mathrm{E}+03$ & $-1,25 \mathrm{E}+05$ & $8,77 \mathrm{E}+04$ & $3,85 \mathrm{E}+04$ & $-1,80 \mathrm{E}-01$ & $5,52 \mathrm{E}-01$ \\
$\mathrm{y}$ (ise) & $2,30 \mathrm{E}+04$ & $6,66 \mathrm{E}+04$ & $-9,69 \mathrm{E}+05$ & $5,22 \mathrm{E}+05$ & $2,60 \mathrm{E}+05$ & $-1,67 \mathrm{E}+00$ & $4,28 \mathrm{E}+00$ \\
$\mathrm{y}$ (is) & $8,44 \mathrm{E}+03$ & $3,33 \mathrm{E}+03$ & $-3,19 \mathrm{E}+05$ & $2,66 \mathrm{E}+05$ & $1,08 \mathrm{E}+05$ & $-1,94 \mathrm{E}-01$ & $1,18 \mathrm{E}+00$ \\
\hline & & & & & & & \\
\hline
\end{tabular}

Fonte: Elaborado pelos autores.

As estatísticas descritivas apresentadas na Tabela 2 mostram que em todos os Estados há variação média positiva do dispêndio com o PBF entre $\mathrm{R} \$ 682.000,00$ (Centro-Oeste) e $\mathrm{R} \$ 6.070 .000$ (Nordeste), evidenciando que o dispêndio aumenta substancialmente a cada mês. As regiões Centro-Oeste e Nordeste também apresentam o menor e o maior desvio-padrão, respectivamente. $\mathrm{O}$ dispêndio com o PBF demonstra também elevada assimetria positiva, ocasionada pela maior probabilidade de variações positivas do que negativas. Também há elevada curtose.

Similarmente, percebe-se considerável aumento médio no consumo de energia, tanto industrial quanto residencial, especialmente na Região Sudeste. A Região Sudeste apresenta o maior desvio-padrão de consumo, seguido da Região Sul, a qual tem o consumo condicionado aos padrões climáticos.

A Tabela 3 apresenta os coeficientes gerados pelas regressões, em que o consumo de energia elétrica residencial foi explicado pelo dispêndio com o PBF. 
Tabela 3 - Consumo de energia elétrica residencial sendo explicado pela despesa com bolsa família

\begin{tabular}{|c|c|c|c|c|c|c|c|}
\hline & $\begin{array}{l}\text { Coeficiente } \\
\text { (símbolo) }\end{array}$ & $\begin{array}{l}\text { Coeficien- } \\
\text { te (valor) }\end{array}$ & $\begin{array}{l}\text { Desvio-Pa- } \\
\text { drão Ro- } \\
\text { busto }\end{array}$ & Teste $\mathrm{t}$ & P-valor & $\mathrm{R}^{2}$ & VIF \\
\hline \multirow{4}{*}{ Brasil } & $\beta_{1}$ & $-0,3133$ & 0,1077 & $-2,91$ & 0,0050 & \multirow{4}{*}{0,1157} & \multirow{4}{*}{3,24} \\
\hline & $\beta_{2}$ & $-0,0027$ & 0,0038 & $-0,70$ & 0,4840 & & \\
\hline & $\beta_{3}$ & 0,0000 & 0,0000 & 0,98 & 0,3300 & & \\
\hline & $\alpha$ & 66733 & 51001 & 1,31 & 0,1950 & & \\
\hline \multirow{4}{*}{$\begin{array}{l}\text { Centro- } \\
\text {-Oeste }\end{array}$} & $\beta_{1}$ & $-0,2119$ & 0,1220 & $-1,74$ & 0,0860 & \multirow{4}{*}{0,0465} & \multirow{4}{*}{3,15} \\
\hline & $\beta_{2}$ & 0,0009 & 0,0036 & 0,24 & 0,8140 & & \\
\hline & $\beta_{3}$ & 0,0000 & 0,0000 & $-0,39$ & 0,6950 & & \\
\hline & $\alpha$ & 3621752 & 2889069 & 1,25 & 0,2140 & & \\
\hline \multirow{4}{*}{ Nordeste } & $\beta_{1}$ & $-0,0499$ & 0,1059 & $-0,47$ & 0,6390 & \multirow{4}{*}{0,0043} & \multirow{4}{*}{3,56} \\
\hline & $\beta_{2}$ & $-0,0003$ & 0,0010 & $-0,27$ & 0,7900 & & \\
\hline & $\beta_{3}$ & 0,0000 & 0,0000 & 0,19 & 0,8480 & & \\
\hline & $\alpha$ & 8886491 & 5947666 & 1,49 & 0,1390 & & \\
\hline \multirow{4}{*}{ Norte } & $\beta_{1}$ & 0,0651 & 0,0862 & 0,76 & 0,4520 & \multirow{4}{*}{0,0535} & \multirow{4}{*}{4,34} \\
\hline & $\beta_{2}$ & 0,0000 & 0,0013 & $-0,01$ & 0,9920 & & \\
\hline & $\beta_{3}$ & 0,0000 & 0,0000 & 1,06 & 0,2920 & & \\
\hline & $\alpha$ & 1653042 & 2076036 & 0,80 & 0,4280 & & \\
\hline \multirow{4}{*}{ Sul } & $\beta_{1}$ & $-0,2684$ & 0,0954 & $-2,81$ & 0,0060 & \multirow{4}{*}{0,1037} & \multirow{4}{*}{2,61} \\
\hline & $\beta_{2}$ & $-0,0060$ & 0,0065 & $-0,93$ & 0,3570 & & \\
\hline & $\beta_{3}$ & 0,0000 & 0,0000 & 1,63 & 0,1070 & & \\
\hline & $\alpha$ & 1682876 & 6712521 & 0,25 & 0,8030 & & \\
\hline \multirow{4}{*}{ Sudeste } & $\beta_{1}$ & $-0,3409$ & 0,1077 & $-3,17$ & 0,0020 & \multirow{4}{*}{0,1267} & \multirow{4}{*}{2,29} \\
\hline & $\beta_{2}$ & $-0,0026$ & 0,0048 & $-0,54$ & 0,5930 & & \\
\hline & $\beta_{3}$ & 0,0000 & 0,0000 & 0,75 & 0,4570 & & \\
\hline & $\alpha$ & 19882 & 18770 & 1,06 & 0,2930 & & \\
\hline
\end{tabular}

Fonte: Elaborado pelos autores. Os valores inferiores a 0,05 foram destacados em negrito. 
A Tabela 3 evidencia que a variação do dispêndio com o PBF não foi capaz de explicar a variação do consumo de energia elétrica em nenhuma região brasileira. As únicas duas variáveis capazes de gerar coeficiente significativo foram os vetores autorregressivos das estimações referentes às regiões Sul e Sudeste e ao Brasil como um todo. Nestas estimações, o coeficiente $\mathrm{R}^{2}$ evidenciou que entre $10,37 \%$ e $12,67 \%$ da variância foi explicada. O fator de inflacionamento da variância esteve dentro dos parâmetros aceitáveis, conforme Gujarati (2009). É possível compreender, portanto, que o consumo de energia elétrica não pode ser explicado pelo dispêndio com PBF.

A Tabela 4 dá seguimento às análises, trazendo os coeficientes estimados pela regressão linear em que o consumo de energia elétrica industrial foi explicado pelo dispêndio com o PBF.

Tabela 4 - Consumo de Energia Elétrica Industrial sendo explicado pela despesa com bolsa família

\begin{tabular}{c|c|r|r|r|r|l|l}
\hline Região & $\begin{array}{c}\text { Coefi- } \\
\text { ciente } \\
\text { (símbolo) }\end{array}$ & $\begin{array}{c}\text { Coefi- } \\
\text { ciente } \\
\text { (valor) }\end{array}$ & $\begin{array}{r}\text { Desvio- } \\
\text {-Padrão } \\
\text { Robusto }\end{array}$ & Teste t & P-valor & $\mathrm{R}^{2}$ & VIF \\
\hline \multirow{3}{*}{ Brasil } & $\beta_{I}$ & 0,2510 & 0,2231 & 1,13 & 0,2640 & & \\
& $\beta_{2}$ & 0,0062 & 0,0067 & 0,92 & 0,3600 & 0,0938 & 3,26 \\
& $\beta_{3}$ & 0,0000 & 0,0000 & $-0,46$ & 0,6450 & & \\
\hline \multirow{3}{*}{ Centro- } & $\alpha$ & -7897156 & 92845 & $-0,09$ & 0,9320 & & \\
-Oeste & $\beta_{1}$ & $-0,0797$ & 0,1261 & $-0,63$ & 0,5290 & & \\
& $\beta_{3}$ & 0,0023 & 0,0038 & 0,61 & 0,5470 & 0,0179 & 3,16 \\
& $\alpha$ & 1135344 & 3227354 & 0,35 & 0,7260 & & \\
Nordes- & $\beta_{I}$ & $-0,4463$ & 0,1081 & $-4,13$ & $\mathbf{0 , 0 0 0 0}$ & & \\
te & $\beta_{2}$ & 0,0013 & 0,0011 & 1,17 & 0,2470 & \multirow{2}{*}{0,2132} & 3,59 \\
& $\beta_{3}$ & 0,0000 & 0,0000 & $-0,45$ & 0,6550 & & \\
\hline
\end{tabular}




\begin{tabular}{c|c|r|r|r|r|l|l}
\hline & $\beta_{I}$ & $-0,4782$ & 0,0946 & $-5,05$ & $\mathbf{0 , 0 0 0 0}$ & & \\
ee_in- & $\beta_{2}$ & 0,0003 & 0,0029 & 0,09 & 0,9310 & 0,2310 & \multirow{2}{*}{4,34} \\
d_n & $\beta_{3}$ & 0,0000 & 0,0000 & $-0,10$ & 0,9230 & & \\
& $\alpha$ & 5876316 & 4801 & 1,22 & 0,2250 & & \\
\hline \multirow{5}{*}{ Sul } & $\beta_{I}$ & $-0,0341$ & 0,1870 & $-0,18$ & 0,8560 & & \\
& $\beta_{2}$ & $-0,0013$ & 0,0128 & $-0,10$ & 0,9210 & 0,0036 & \multirow{2}{*}{2,56} \\
& $\beta_{3}$ & 0,0000 & 0,0000 & $-0,12$ & 0,9070 & & \\
& $\alpha$ & 9570459 & 14610 & 0,66 & 0,5140 & & \\
\hline \multirow{5}{*}{ Sudeste } & $\beta_{1}$ & 0,1477 & 0,2224 & 0,66 & 0,5090 & & \\
& $\beta_{2}$ & 0,0065 & 0,0092 & 0,71 & 0,4780 & \multirow{2}{*}{0,0445} & 2,34 \\
& $\beta_{3}$ & 0,0000 & 0,0000 & $-0,20$ & 0,8400 & & \\
& $\alpha$ & 2205377 & 36527 & 0,06 & 0,9520 & & \\
\hline
\end{tabular}

Fonte: Elaborado pelos autores.

Os resultados da Tabela 4 corroboram o que foi verificado na Tabela 3, pois os únicos coeficientes significativos, ao grau de significância de 5\%, foram gerados pelo vetor autorregressivo. Estes coeficientes são negativos, de maneira semelhante à verificada na Tabela 3 , mas aparecem nas regiões Norte e Nordeste. Assim, a variação do consumo de energia elétrica industrial é explicada por seu vetor autorregressivo em $t-1$ nas regiões Norte e Nordeste, ao passo que a energia elétrica residencial é explicada nas regiões Sul, Sudeste e para o Brasil como um todo. Nas regressões referentes às regiões Norte e Nordeste, o coeficiente $\mathrm{R}^{2}$ evidencia que 21,32\% e 23,10\%, respectivamente, da variância da energia elétrica industrial é explicada.

Novamente percebe-se que o fator de inflacionamento da variância ficou dentro dos parâmetros aceitáveis em todas as regressões. A variação do dispêndio com o PBF não explicou a variação do consumo de energia elétrica industrial. 
No intuito de aprofundar a análise, a Tabela 5 traz o resultado dos testes de causalidade de Granger, os quais evidenciarão se há indícios de uma relação de causa e efeito entre as variáveis.

Tabela 5 - Testes de causalidade

\begin{tabular}{|c|c|c|c|}
\hline Variáveis relacionadas & $\operatorname{chi}^{2}$ & GL & p-valor \\
\hline bf_brasil & 2,144 & 2 & 0,342 \\
\hline ee_res_brasil & 0,556 & 2 & 0,757 \\
\hline bf_co & 0,994 & 2 & 0,608 \\
\hline ee_res_co & 4,216 & 2 & 0,122 \\
\hline bf_ne & 0,322 & 2 & 0,851 \\
\hline ee_res_ne & 0,835 & 2 & 0,659 \\
\hline bf_n & 1,495 & 2 & 0,474 \\
\hline ee_res_n & 3,496 & 2 & 0,174 \\
\hline bf_s & 4,423 & 2 & 0,110 \\
\hline ee_res_s & 0,450 & 2 & 0,798 \\
\hline bf_se & 3,742 & 2 & 0,154 \\
\hline ee_res_se & 0,362 & 2 & 0,834 \\
\hline bf_brasil & 0,551 & 2 & 0,759 \\
\hline ee_ind_brasil & 0,583 & 2 & 0,747 \\
\hline bf_co & 1,024 & 2 & 0,599 \\
\hline ee_ind_co & 0,391 & 2 & 0,822 \\
\hline bf_ne & 1,219 & 2 & 0,544 \\
\hline ee_ind_ne & 0,399 & 2 & 0,819 \\
\hline bf_n & 0,947 & 2 & 0,623 \\
\hline ee_ind_n & 0,893 & 2 & 0,640 \\
\hline bf_s & 0,014 & 2 & 0,993 \\
\hline ee_ind_s & 1,809 & 2 & 0,405 \\
\hline bf_se & 0,203 & 2 & 0,904 \\
\hline ee_ind_se & 0,658 & 2 & 0,720 \\
\hline
\end{tabular}

Fonte: Elaborado pelos autores. 
A Tabela 5 aponta que não há indícios de relações de causalidade entre as variáveis referentes ao dispêndio com o PBF e as variáveis referentes ao consumo de energia elétrica, tanto industrial quanto residencial. Estes resultados corroboram os encontrados nas Tabelas anteriores, no sentido que não há evidências de que o dispêndio com o PBF afeta o crescimento econômico do ponto de vista macro.

\section{Conclusões}

A frequente avaliação das políticas e programas sociais se faz necessária para que se tenham indicadores que ajudem a apresentar resultados que, de acordo com as estratégias de governo, tomem medidas de ajuste sobre o impacto econômico-social que causam. A utilização das práticas de avaliação e monitoramento permite à administração pública federal ajustar o desempenho do programa.

As estatísticas descritivas apresentadas na Tabela 2 mostram que em todos os Estados há variação média positiva do dispêndio com o PBF, e nas regiões Centro-Oeste e Nordeste há aumento no dispêndio a cada mês estudado. O dispêndio com o PBF tem também elevada assimetria positiva, ocasionada pela maior probabilidade de variações positivas do que negativas.

Similarmente, percebe-se considerável aumento médio no consumo de energia tanto industrial quanto residencial, especialmente na Região Sudeste. A Região Sudeste apresenta o maior desvio-padrão de consumo, seguida da Região Sul, que tem o consumo condicionado aos padrões climáticos.

Assim, a variação do consumo de energia elétrica industrial é explicada nas regiões Norte e Nordeste, ao passo que a energia elétrica residencial é explicada nas regiões Sul, Sudeste e para o Brasil como um todo. Os resultados mostram que não há indícios de relações de causalidade entre as variáveis referentes ao dispêndio com o PBF e as variáveis referentes ao consumo 
de energia elétrica, tanto industrial quanto residencial; sendo assim, não há evidências de que o dispêndio com o PBF afeta o crescimento econômico do ponto de vista macroeconômico.

Apesar de o Programa Bolsa Família não apresentar impacto relevante no que se refere ao desenvolvimento econômico, é claro que a transferência de renda, principal motivo da criação do programa, é impactada de forma significativa no desenvolvimento social, causando uma grande diferença na vida de muitas famílias brasileiras, trazendo redução das desigualdades sociais.

O efeito a longo prazo que o PBF traz às famílias em situação de extrema pobreza é de suma importância no aspecto social, apesar de não apresentar causalidade entre as variáveis referentes ao dispêndio com o Programa e as variáveis referentes ao consumo de energia elétrica das regiões brasileiras. Vale ressaltar que muitos possuem acesso à energia clandestina, o que pode trazer uma distorção na análise dos dados.

Concluímos que se o PBF não contribui para o crescimento econômico ele muda a realidade e expectativa de vida de muitas famílias, e somente por esse motivo se justifica, tendo resultados no aumento da frequência escolar, um dos critérios para ter direito a receber o benefício. Com isso, o $\mathrm{PBF}$ combate à pobreza e dá acesso aos direitos sociais da população mais vulnerável do país.

\section{Referências}

BRASIL. Pesquisa Nacional por Amostra de Domicílio (PNAD), 2004, 2005, 2006. GUJARATI, D. Econometria básica. São Paulo: Makron Books, 2009.

GUSMÃO, G. C. Impacto do Programa Bolsa Família no município de São João da Ponte - MG. 2009. Dissertação (Mestrado em Economia) - Universidade Federal de Viçosa, Viçosa, MG, 2009. 
HOFFMANN, R. Desigualdade da renda e das despesas per capita no Brasil, em 2002-2003 e 2008-2009, e avaliação do grau de progressividade ou regressividade de parcelas da renda familiar. Revista Economia e Sociedade, Campinas, v. 19, n. 3 (40), p. 647-661, dez. 2010.

LICIO, E. C.; MESQUITA, C. S.; CURRALERO, C. R. B. Desafios para a Coordenação Intergovernamental do Programa Bolsa Família. Revista de Administração de Empresas, São Paulo, vol. 51, n. 5, 2011.

MEDEIROS, M.; BRITTO, T.; SOARES, F. Programas focalizados de transferências de renda no Brasil: contribuições para o debate. Brasília: IPEA, 2007. (Texto para Discussão ${ }^{\circ} 1.283$ ).

MELO, R. M. S.; DUARTE, G. B. Impacto do Programa Bolsa Família sobre a Frequência Escolar: o caso da agricultura familiar no Nordeste do Brasil. Revista de Economia e Sociologia Rural, Piracicaba, SP, vol. 48, n. 3, p. 635-656, jul./set. 2010.

MINISTÉRIO DO DESENVOLVIMENTO SOCIAL. MDS. 2011. MDS em Números. Disponível em: <http://aplicacoes.mds.gov.br/sagi/ascom/index.php?cu$\mathrm{t}=\mathrm{aHR} 0 \mathrm{cDovL2F}$ wbGljYWNvZXMubWRzLmdvdi5ici9zYWdpL2FzY29tL2dlcmFyL2luZGV4LnBocA==\&def=v>. Acesso em: 5 mar. 2013.

MOURA, P. G. M. Bolsa Família: projeto social ou marketing político? Revista Katálogo, Florianópolis, v. 10, n. 1, p. 115-122, jan./jun. 2007.

REGO, W. L. Aspectos teóricos das políticas de cidadania: uma aproximação ao Bolsa Família. Revista Lua Nova, São Paulo, n. 73, p. 147-185, 2007.

ROCHA, S. O Programa Bolsa Família: evolução e efeitos sobre a pobreza. Revista Economia e Sociedade, Campinas, SP, vol. 20, n. 1, p. 113-139, 2011.

SANTOS, G. G. et al. Mercado de trabalho e rendimento no meio rural brasileiro. Revista Economia Aplicada, São Paulo, vol. 14, n. 3, 2010.

SANTOS, S. M. C.; SANTOS, L. M. P. Avaliação de políticas públicas de segurança alimentar e combate à fome no período de 1995-2002. 1 - Abordagem metodológica. Cad. Saúde Pública, Rio de Janeiro, 23(5):1.029-1.040, maio 2007.

Recebido em: 20/12/2015

Aceito em: $1 \% 6 / 2016$ 